\title{
NUEVAS ADICIONES A LA FLORA INTRODUCIDA DE CHILE
}

\author{
Joel Calvo \& Andrés Moreira-Muñoz
}

Instituto de Geografí, Facultad de Ciencias del Mar y Geografía, Pontificia Universidad Católica de Valparaíso, Avenida Brasil 2241,2362807 Valparaíso, Chile; calvocasas@gmail.com (autor corresponsal).

\begin{abstract}
Calvo, J. \& A. Moreira-Muñoz. 2018. New additions to the exotic flora of Chile. Darwiniana, nueva serie 6(2): 179-185.

Fumaria muralis (Papaveraceae) and Lathyrus latifolius (Leguminosae) are recorded for the first time in Chile as alien species. Detailed pictures of both species are provided, together with keys to the species of Fumaria and Lathyrus in Chile and a map with the location of the new records.
\end{abstract}

Keywords. Chile; exotic flora; Fumaria; Lathyrus; Valparaíso.

Resumen. Calvo, J. \& A. Moreira-Muñoz. 2018. Nuevas adiciones a la flora introducida de Chile. Darwiniana, nueva serie 6(2): 179-185.

Las especies alóctonas Fumaria muralis (Papaveraceae) y Lathyrus latifolius (Leguminosae) se registran por primera vez en Chile. Se aportan fotografías detalladas de ambas especies, junto con claves para distinguir a las especies de Fumaria y de Lathyrus presentes en Chile, y un mapa con la localización de los nuevos registros.

Palabras clave. Chile; flora introducida; Fumaria; Lathyrus; Valparaíso.

\section{INTRODUCCIÓN}

Estudios recientes estiman en 743 el número de especies introducidas (o exóticas) y naturalizadas en Chile, lo que representa alrededor del 15\% de la flora chilena (Fuentes et al., 2013, 2014; Rodríguez et al., 2018). Por lo que refiere a la ciudad de Santiago de Chile, se calcula que el $85,1 \%$ de los 508 táxones que se registraron en los espacios públicos corresponden a especies introducidas (Figueroa et al., 2016). Fuentes et al. (2014) apuntan que gran parte de ellas llegaron al país en la época de la colonización española; sin embargo, no es baladí el número de introducciones que tuvo lugar durante los siglos XIX y XX debido a la llegada de nuevos inmigrantes y la internacionalización de Chile.
La mayoría de las especies proceden de Europa y Norteamérica, y donde mejor han medrado es en las regiones de clima mediterráneo y templado del país, i.e., centro-sur de Chile (Fuentes et al., 2013). Por ejemplo, en el Parque Nacional La Campana (Región de Valparaíso), cerca del 93\% de las especies ruderales introducidas procede de Europa mediterránea (Hauck et al., 2016). Cabe mencionar, además, que en los últimos años se han detectado nuevas especies que se deberían añadir a las 743 estimadas en 2014, e.g., Carduus tenuiflorus Curtis, Dittrichia viscosa (L.) Greuter, Ehrharta longiflora Sm., Euphorbia terracina L.; detalles para cada una de estas especies pueden encontrarse en Cordero et al. (2016), Novoa (2017), Calvo \& Moreira-Muñoz (2018), MacayaBerti \& Teillier (2017), respectivamente. 
En este trabajo se registra por primera vez a Fumaria muralis Sond. ex W. D. J. Koch (Papaveraceae) y Lathyrus latifolius L. (Leguminosae) como especies introducidas en Chile. El género Fumaria L. contiene ca. 50 especies nativas de Eurasia, África y Macaronesia, con su mayor diversidad en la región mediterránea occidental (Boufford, 1997). En Chile, hasta el momento, se habían reportado Fumaria agraria Lag., F. capreolata L., F. officinalis L. y F. parviflora Lam. (Marticorena \& Quezada, 1985; Matthei, 1995; Lidén, 2008; Rodríguez et al., 2018). Zöllner et al. (1995) también citaron a Fumaria densiflora DC. y F. rostellata Knaf para la Región de Valparaíso, pero su presencia no ha sido confirmada. Por lo que refiere al género Lathyrus L., comprende alrededor de 160 especies mayormente distribuidas en los climas templados del hemisferio norte, con su centro de diversidad en la región mediterránea oriental. En Chile son tres las especies introducidas detectadas hasta la fecha: Lathyrus cicera L., L. japonicus Willd. y L. sativus L. (Marticorena \& Quezada, 1985; Fortunato, 2008; Rodríguez et al., 2018; Tropicos.org, 2018). La presencia de Lathyrus hirsutus L. en Chile es dudosa (Fortunato, 2008). En las claves dicotómicas que se aportan más adelante no se han incluido las especies cuya presencia es dudosa en Chile.

Los nuevos hallazgos han sido posibles gracias a periódicas campañas de recolección llevadas a cabo en Valparaíso y sus áreas periurbanas. El conocimiento de la flora introducida de una región es esencial para el manejo adecuado de ésta en aras de preservar los ecosistemas naturales (Fuentes et al., 2013). Aunque Chile goza de un conocimiento notable de su flora introducida y naturalizada, esta contribución pone de manifiesto que todavía son necesarios mayores esfuerzos para completarlo.

\section{MATERIALES Y MÉTODOS}

Las recolecciones llevadas a cabo durante los años 2017-2018 en la ciudad de Valparaíso y en sus áreas periurbanas han permitido localizar los dos nuevos registros que se comunican. Los especímenes se han identificado mediante el uso de las claves de la Flora iberica, realizadas por Lidén (1986) en el caso de Fumaria, y por Gallego (1999) para el de Lathyrus; estas claves se han adaptado para la identificación de las especies introducidas en Chile. El material recolectado fue comparado con el de las colecciones que alberga el herbario MA y depositado en CONC y SGO (Thiers, 2018).

\section{RESULTADOS}

Fumaria muralis Sond. ex W.D.J. Koch, Syn. Fl. Germ. Helv. ed. 2: 1017. 1845. TIPO: Alemania, "Hamburg", VII-1844, $W$. Sonder s.n. (lectotipo MEL cb 1584466 foto! designado por Walsh, Muelleria 7: 496. 1992). Figs. 1-2.

Hierba anual, difusa o trepadora. Hojas con los segmentos ovados a linear-lanceolados, generalmente de anchura mayor de 1,5 mm. Racimos de la misma longitud o mayores que su pedúnculo. Pedicelos fructíferos erectopatentes, raramente algo arqueados. Flores con los sépalos orbicular-ovados, dentados; corola de (8)9-11 mm, generalmente rosada, con las alas del pétalo superior de color púrpura oscuro. Fruto subgloboso a ovoide, finamente ruguloso o casi liso.

Referencias. Para una descripción detallada de la especie véase Lidén (1986: 459).

Iconografía. Véase Valdés (1987: 145).

Distribución y hábitat. Fumaria muralis se considera nativa del oeste de Europa y Macaronesia (Lidén, 1986). No obstante, se encuentra bien extendida por otras regiones como especie introducida; se ha reportado en Australia, California, Nueva Zelanda y Sudáfrica. En Chile se la encontró en Valparaíso (Fig. 3), en el cerro San Juan de Dios, donde crece en varios terrenos yermos y sobre muros sombríos. Como especies acompañantes destacamos a Fumaria capreolata y Parietaria judaica L. Este registro representa la primera cita en Sudamérica de esta especie. 


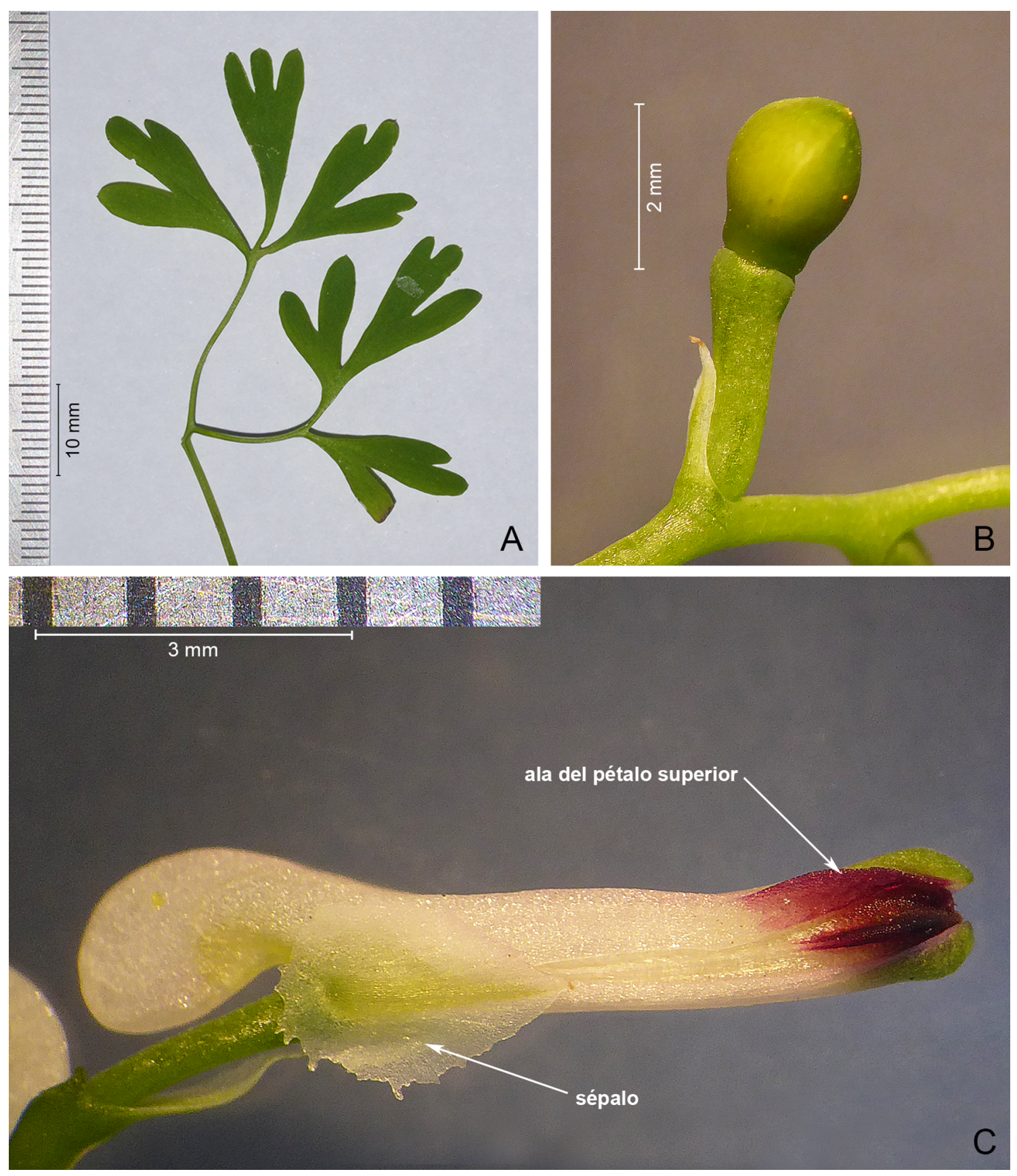

Fig. 1. Fumaria muralis (Calvo 7733). A, detalle de los segmentos foliares. B, detalle del fruto. C, detalle de la flor. Fotografías de J. Calvo. Figura en color en la versión en línea http://www.ojs.darwin.edu.ar/index.php/darwiniana/article/view/801/780

Observaciones. Fumaria muralis se confunde con $F$. agraria por la anchura de sus segmentos foliares, por lo general mayores de $1,5 \mathrm{~mm}$ en ambas especies (Fig. 1A). No obstante, caracteres relativos al pétalo superior de las flores, a los sépalos y al fruto nos permiten separarlas con cierta facilidad. Fumaria muralis tiene las alas del pétalo superior de 


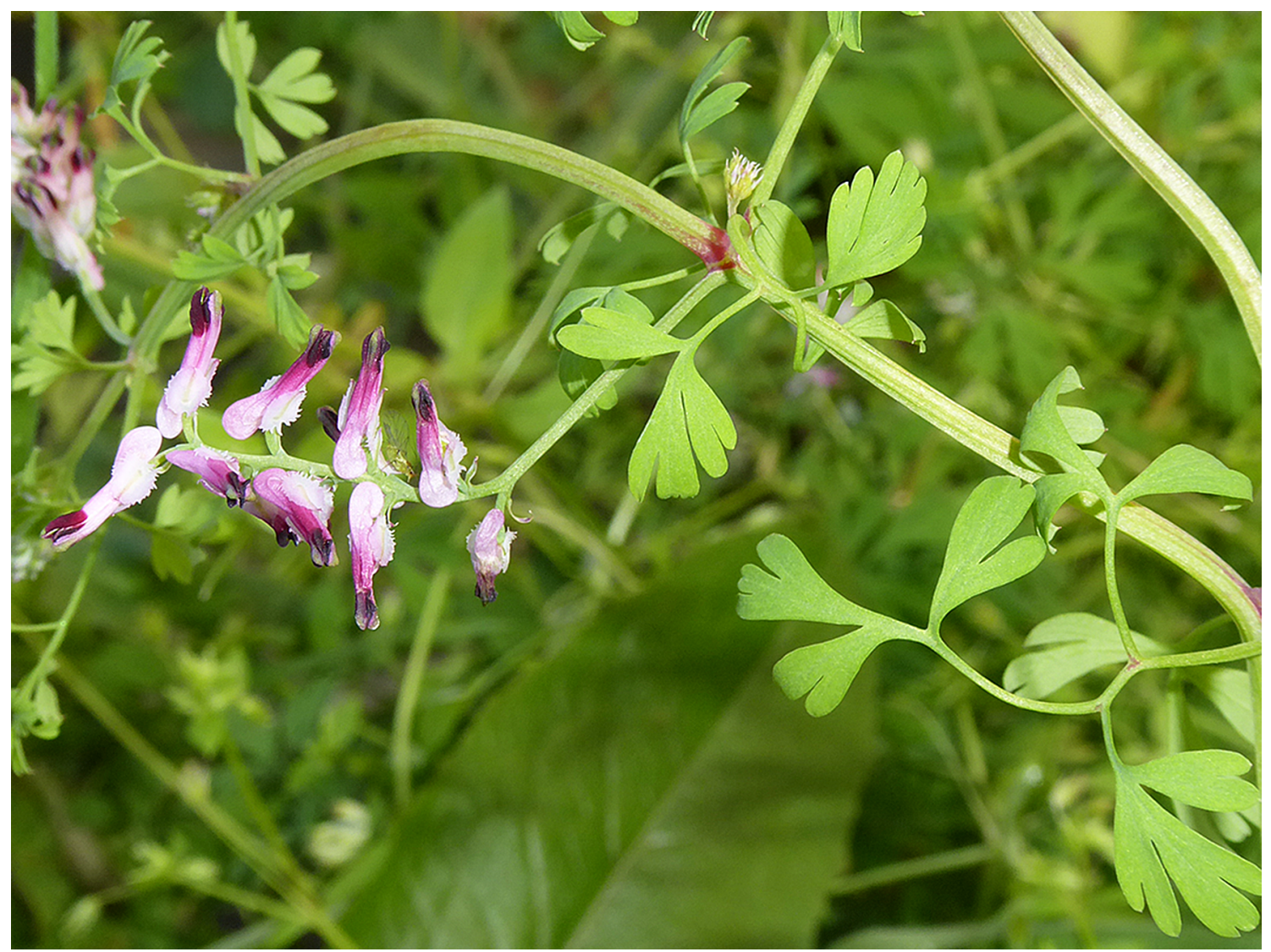

Fig. 2. Fumaria muralis (Calvo 7574). Hábito. Fotografía de J. Calvo. Figura en color en la versión en línea http:// www.ojs.darwin.edu.ar/index.php/darwiniana/article/view/801/780

color púrpura oscuro, distinto al color rosado (o blanquecino) del resto del pétalo (Fig. 1C); por el contrario, en F. agraria todo el pétalo es de color uniforme (de rosado a blanquecino). Para estudiar este carácter es importante distinguir bien las alas del pétalo superior de los pétalos interiores, que, en F. agraria, también presentan el ápice púrpura oscuro. Por lo que refiere a los sépalos, estos son dentados en $F$. muralis y subenteros en F. agraria. Por último, el fruto es mucho más liso en F. muralis (Fig. 1B).

En Valparaíso se la ha observado creciendo junto a $F$. capreolata, esta última muy extendida y abundante en muros y sitios sombríos de los cerros. Su confusión es poco probable porque $F$. capreolata presenta pedicelos fructíferos fuertemente recurvados o reflejos y sépalos muy anchos, a menudo más que la anchura de las flores.

Aunque la especie nunca había sido citada para Chile (Marticorena \& Quezada, 1985; Matthei, 1995; Lidén, 2008; Rodríguez et al., 2018), cabe mencionar que en el herbario MO hay un pliego recolectado en 1965 cerca de Viña del Mar (El Salto) y determinado como $F$. muralis (MO 2199786). Desafortunadamente no hemos podido estudiar dicho material.

\section{Material representativo examinado}

CHILE. Región de Valparaíso. Prov. Valparaíso, Valparaíso, cerro San Juan de Dios, $33^{\circ} 02^{\prime} \mathrm{S} 71^{\circ} 37^{\prime} \mathrm{W}, 80-120$ m s. m., 10IX-2017, Calvo 7574 (CONC, SGO); 4-VI2018, Calvo 7733 (CONC). 


\section{Clave para la identificación de las especies de Fumaria en Chile}

1. Pedicelos fructíferos fuertemente recurvados o reflejos F. capreolata

1. Pedicelos fructíferos erectos o erecto-patentes, raramente algo arqueados

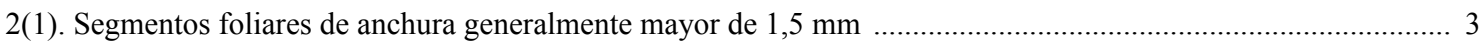

2. Segmentos foliares de anchura generalmente menor de $1,5 \mathrm{~mm}$

3(2). Pétalo superior de color más o menos uniforme; sépalos subenteros; fruto tuberculado-rugoso

F. agraria

3. Pétalo superior con alas de color púrpura oscuro; sépalos dentados; fruto ruguloso o casi liso F. muralis 4(2). Sépalos mayores de $1,5 \mathrm{~mm}$; fruto más ancho que largo F. officinalis 4. Sépalos igual o menores de $1 \mathrm{~mm}$; fruto tan ancho como largo F. parviflora

Lathyrus latifolius L., Sp. Pl.: 733. 1753. TIPO: Herb. Clifford: 367, Lathyrus 7 (lectotipo BM cb 000646673! designado por Lassen en Turland \& Jarvis, Taxon 46: 474. 1997). Fig. 4.

Hierba perenne rizomatosa, glabra, con tallos trepadores de hasta $2 \mathrm{~m}$, alados. Hojas generalmente con un solo par de folíolos, terminadas en un zarcillo ramificado y con estípulas en la base. Folíolos de tamaño y forma muy variable, de oblanceolados a linearlanceolados. Inflorescencias pedunculadas con (2)3-12 flores. Cáliz con dientes desiguales, los superiores menores o de igual longitud que el tubo; corola papilionada, de (15)18-26(34) mm, roja o purpúrea, a menudo con las alas más oscuras. Legumbre de 6-9 cm, glabra, con 13-22 semillas.

Referencias. para una descripción detallada de la especie véase Gallego (1999: 442).

Iconografía. Véase Gallego (1999: 443, fig. 118 d-i).

Distribución y hábitat. Especie nativa del centro, sur y este de Europa, norte de África y de las islas Canarias (Gallego, 1999). Introducida en Argentina, Australia, Canadá, China, Estados Unidos (incluyendo Hawái), Sudáfrica, Uruguay, entre otros. En Chile, fue encontrada en una ladera de la quebrada de Jaime en Valparaíso y en unos taludes en la entrada de Viña del Mar (Fig. 3), junto a otras especies naturalizadas y a especies nativas. Entre las nativas se destacan Lobelia polyphylla Hook. \& Arn. y Oxalis rosea Jacq., y entre las naturalizadas a Senecio angulatus L.f. y Spartium junceum L.
Observaciones. Lathyrus latifolius sobresale entre las especies del género naturalizadas en Chile por sus tallos alados de hasta $2 \mathrm{~m}$ de longitud; L. japonicus es otra especie también

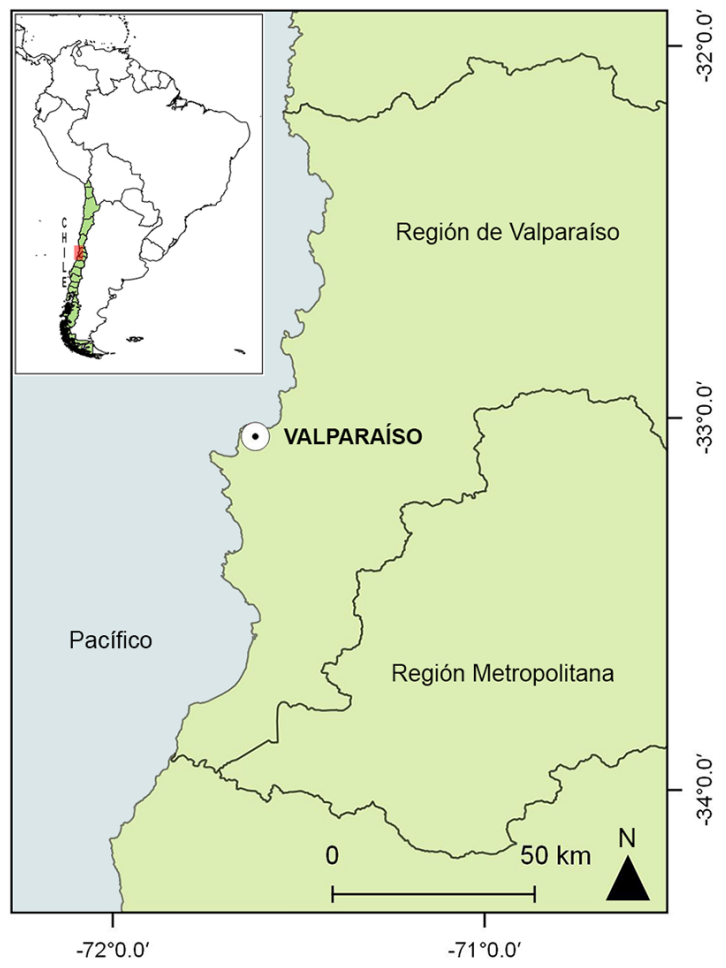

Fig. 3. Área de recolección de Fumaria muralis y Lathyrus latifolius en Chile (Valparaíso, Región de Valparaíso). Figura en color en la versión en línea http://www.ojs. darwin.edu.ar/index.php/darwiniana/article/view/801/780 
robusta, pero sus tallos son angulados en lugar de alados. Además, las hojas tienen de 6-10 folíolos, mientras que L. latifolius por lo general solamente desarrolla 2 folíolos (Fig. 4A). Sus grandes y llamativas flores son también características, así como su fruto de $6-9 \mathrm{~cm}$, glabro (Fig. 4B). Las alas de la flor (pétalos laterales) generalmente son más oscuras o del mismo color que el resto de pétalos, mientras que en L. japonicus las alas suelen ser más claras.
Las otras especies son más humildes y con flores menores.

\section{Material representativo examinado}

CHILE. Región de Valparaíso. Prov. Valparaíso, Valparaíso, quebrada de Jaime, $33^{\circ} 03^{\prime} \mathrm{S} 71^{\circ} 36^{\prime} \mathrm{W}$, 120 m s. m., 3-X-2017, Calvo 7591 (CONC, SGO); Viña del Mar, Av. España, entre calle Valparaíso y Reloj de Flores, $33^{\circ} 01^{\prime}$ 'S $71^{\circ} 33^{\prime} \mathrm{W}, 30 \mathrm{~m}$ s. m., 1-X2018, Calvo 7788 (SGO).

\section{Clave para la identificación de las especies introducidas de Lathyrus en Chile}

1. Tallo angulado; estípulas casi del mismo tamaño que los folíolos; folíolos a menudo crasos

L.japonicus

1. Tallo alado; estípulas más pequeñas que los folíolos; folíolos no crasos

2(1). Lóbulos superiores del cáliz más cortos o de igual longitud que el tubo L. latifolius

2. Lóbulos superiores del cáliz más largos que el tubo

$3(2)$. Fruto con dos quillas ventrales de hasta $0,8 \mathrm{~mm}$ de anchura; quilla con el margen vexilar recto L. cicera

3. Fruto con dos alas ventrales de $(0,8) 1,2-2,7 \mathrm{~mm}$ de anchura; quilla falcada L. sativus
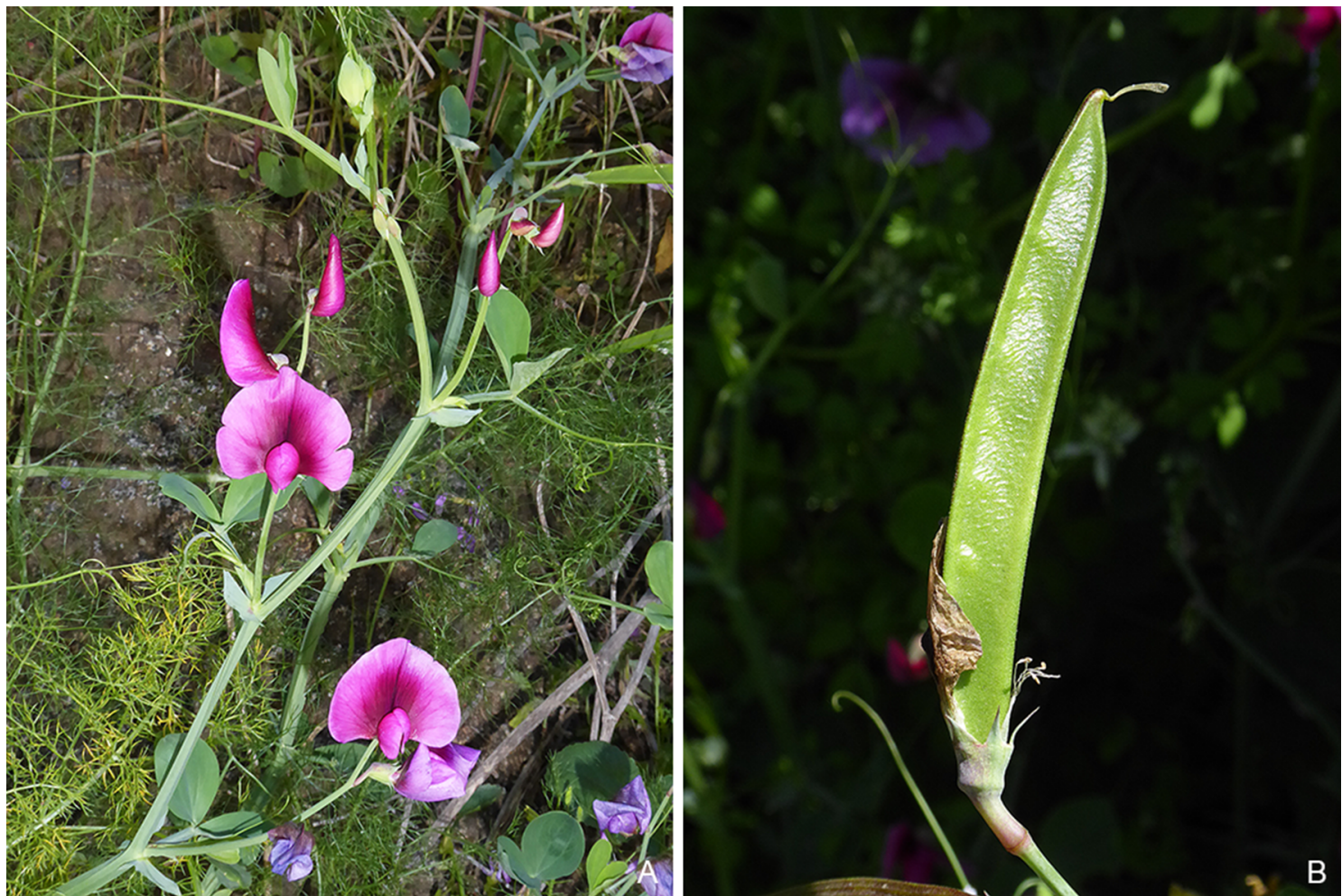

Fig. 4. Lathyrus latifolius (Calvo 7591). A, hábito. B, detalle fruto. Fotografías de J. Calvo. Figura en color en la versión en línea http://www.ojs.darwin.edu.ar/index.php/darwiniana/article/view/801/780 


\section{AGRADECIMIENTOS}

Estamos agradecidos a los conservadores de los herbarios citados en el texto, así como a los revisores anónimos por sus valiosos comentarios. Este trabajo ha sido financiado por FONDECYT, a través de una beca postdoctoral del primer autor (proyecto $\mathrm{N}^{\circ} 3170270$ ).

\section{BIBLIOGRAFÍA}

Boufford, D. E. 1997. Fumaria L., en Flora of North America Editorial Committee, Flora of North America vol. 3: Magnoliophyta: Magnoliidae and Hamamelidae, pp. 356357. New York; Oxford: Oxford University Press.

Calvo, J. \& A. Moreira-Muñoz. 2018. First record of Ehrharta longiflora Sm. (Poaceae, Ehrharteae) for South America. Check List 14: 475-478. DOI: 10.15560/14.2.475

Cordero, S.; D. G. Gutiérrez \& A. Moreira-Muñoz. 2016. Carduus tenuiflorus Curtis (Asteraceae), nuevo registro para la flora alóctona de Chile. Gayana. Botánica 73: 453-456. DOI: $10.4067 / \mathrm{S} 0717-66432016000200453$

Figueroa, J. A.; S. Teillier, N. Guerrero-Leiva, C. Ray-Bobadilla, S. Rivano, D. Saavedra \& S. A. Castro. 2016. Vascular flora in public spaces of Santiago, Chile. Gayana. Botánica 73: 85103. DOI: $10.4067 / \mathrm{S} 0717-66432016000100011$

Fortunato, R. H. 2008. Lathyrus L., en F. O. Zuloaga, O. Morrone \& M. J. Belgrano (eds.), Catálogo de las plantas vasculares del Cono Sur (Argentina, Sur de Brasil, Chile, Paraguay y Uruguay). Monographs in Systematic Botany from the Missouri Botanical Garden 107: 2201-2206.

Fuentes, N.; A. Pauchard, P. Sánchez, J. Esquivel \& A. Marticorena. 2013. A new comprehensive database of alien plant species in Chile based on herbarium records. Biological Invasions 15: 847-858. DOI: 10.1007/s10530-012-0334-6

Fuentes, N.; P. Sánchez, A. Pauchard, J. Urrutia, L. Cavieres \& A. Marticorena. 2014. Plantas Invasoras del Centro-Sur de Chile: Una Guía de Campo. Concepción, Chile: Laboratorio de Invasiones Biológicas (LIB).

Gallego, M. J. 1999. Lathyrus L., en S. Talavera, C. Aedo, S. Castroviejo, C. Romero Zarco, L. Sáez, F. J. Salgueiro \& M. Velayos (eds.), Flora iberica vol. 7(1): Leguminosae (partim), pp. 423-482. Madrid: servicio de publicaciones del C.S.I.C.
Hauck, L.; A. Moreira-Muñoz \& W. Nezadal. 2016. La flora exótica ruderal del Parque Nacional La Campana, Región de Valparaíso, Chile central. Gayana. Botánica 73: 206-219. DOI: 10.4067/S0717-66432016000200206

Lidén, M. 1986. Fumaria L., en S. Castroviejo, M. Laínz, G. López González, P. Montserrat, F. Muñoz Garmendia, J. Paiva \& L. Villar (eds.), Flora iberica vol. 1: Lycopodiaceae-Papaveraceae, pp. 447-467. Madrid: servicio de publicaciones del C.S.I.C.

Lidén, M. 2008. Fumaria L., en F. O. Zuloaga, O. Morrone \& M. J. Belgrano (eds.), Catálogo de las plantas vasculares del Cono Sur (Argentina, Sur de Brasil, Chile, Paraguay y Uruguay). Monographs in Systematic Botany from the Missouri Botanical Garden 107: 2321-2322.

Macaya-Berti, J. \& S. Teillier. 2017. Euphorbia terracina L. (Euphorbiaceae) advena en Chile. Chloris Chilensis Año 20 $\mathrm{N}^{\mathrm{o}}$ 1. http://www.chlorischile.cl/euphorbia\%20terracina\%20 x\%20macaya\%20et\%20al/Euphorbia\%20terracina.htm

Marticorena, C. \& M. Quezada. 1985. Catálogo de la flora vascular de Chile. Gayana. Botánica 42(1-2): 1-157.

Matthei, O. 1995. Manual de las malezas que crecen en Chile. Santiago de Chile: Alfabeta Impresores.

Novoa, P. 2017. Dittrichia viscosa (L.) Greuter(Asteraceae): Nuevo registro para la flora introducida de Chile. Gayana. Botánica 74: 293-295. DOI: 10.4067/S0717-66432017000200293

Rodríguez, R.; C. Marticorena, D. Alarcón, C. Baeza, L. Cavieres, V. L. Finot, N. Fuentes, A. Kiessling, M. Mihoc, A. Pauchard, E. Ruiz, P. Sánchez \& A. Marticorena. 2018. Catálogo de las plantas vasculares de Chile. Gayana. Botánica 75: 1-430. http://www.gayanabotanica.cl/ pdfs/2018/1/01_Rodriguez_etal_2018.pdf

Thiers, B. [permanentemente actualizado, consulta 2018] Index Herbariorum: a global directory of public herbaria and associated staff. New York Botanical Garden's Virtual Herbarium, http://sweetgum.nybg.org/ih [consulta junio 2018].

Tropicos.org. Missouri Botanical Garden. 2018, http://www. tropicos.org [consulta junio 2018].

Valdés, B. 1987. Fumaria L., en B. Valdés, S. Talavera \& E. Fernández-Galiano (eds.), Flora vascular de Andalucía Occidental vol. 1, pp. 140-147. Barcelona: Ketres Editora.

Zöllner, O.; M. Olivares \& M. E. Varas. 1995. El género Fumaria L. (Fumariaceae) en la zona central de Chile. Anales del Museo de Historia Natural de Valparaíso 23: 21 31. http://www.mhnv.cl/636/articles-56743_archivo_01.pdf 